\title{
Kernos
}

Revue internationale et pluridisciplinaire de religion grecque antique

3 | 1990

Varia

\section{Mantique et oracles dans l'oeuvre de Philon d'Alexandrie}

\section{Baudouin Decharneux}

\section{(2) OpenEdition \\ Journals}

\section{Édition électronique}

URL : http://journals.openedition.org/kernos/978

DOI : $10.4000 /$ kernos.978

ISSN : 2034-7871

\section{Éditeur}

Centre international d'étude de la religion grecque antique

\section{Édition imprimée}

Date de publication : 1 janvier 1990

ISSN : 0776-3824

\section{Référence électronique}

Baudouin Decharneux, "Mantique et oracles dans l'oeuvre de Philon d'Alexandrie », Kernos [En ligne], 3 | 1990, mis en ligne le 19 avril 2011, consulté le 30 avril 2019. URL : http://journals.openedition.org/ kernos/978; DOI : 10.4000/kernos.978 


\section{MANTIQUE ET ORACLES \\ DANS L'GEURE DE PHILON D'ALEXANDRIE}

à Anne et Max Wientzen

Au regard des communications précédentes, il nous est permis de circonscrire de manière plus systématique et plus précise la fonction, ou plutôt les fonctions, religieuse, politique, culturelle, voir thérapeutique assumées par les oracles et la mantique dans le monde grec antique.

On notera que si les pratiques oraculaires et divinatoires semblent principalement développées autour du culte d'Apollon, le cadre religieux dans lequel elles apparaissent et se développent demeure essentiellement polythéiste. Il est dès lors intéressant d'analyser la confrontation entre les traditions oraculaires et divinatoires issues du polythéisme grec et celles issues du milieu monothéiste juif d'Alexandrie. À cet égard, Philon s'avère être un témoin privilégié, car la richesse des textes qui concernent la divination tant du point de vue sémantique que philosophique est sans nul doute significative du grand intérêt porté par l'Alexandrin à ces matières ${ }^{1}$. Notre propos s'articule en deux parties :

1 Les principaux textes de Philon en matière de divination et d'oracles sont : Quod Deus sit immutabilis, 180, où Philon qualifie Balaam de rejeton de la Terre et non du Ciel et décrit le déluge interne qui noie le devin attaché aux Oracles et présages mensongers; De confusione linguarum, 159, où l'étymologie hébraïque de Balaam est analysée et définie comme "peuple vain» (voir sur ce point la traduction de J.G. KAHN, De confusione linguarum, édition du Cerf, 1963, p. 130 et plus précisément note 3, dans le De Cherubim, de la même collection, J. GoREZ traduit ton mataion laon par «peuple frivole»). Ce très intéressant passage démontre combien la problématique de la divination est enracinée dans la problématique plus vaste du Logos. Le type de divination pratiquée par Balaam est dans ce passage définie comme «... dénuée d'inspiration authentique»; De Somniis, I, II, naturellement le traité de Philon le plus complet concernant la divination, particulièrement intéressant dans la mesure où il lie la problématique de la divination avec le thème des vertus (Jacob et Joseph); Quis rerum divinarum heres sit, 96, où Philon évoque la divination astrale chez les Chaldéens; De Vita Mosis, 263-295, Philon y développe longuement l'anecdote du devin Balaam, symbole du devin technique non inspiré. En outre Philon nous décrit Balaam d'une manière ne touchant pas directement la divination en De Cherubim, 32-33, où Balaam apparaît comme un homme cupide attaché aux plaisirs et aux biens matériels; en Quod Deterius, 71, où le Devin est qualifié de Sophiste, et en De migratione Abrahami, 114-115, où le thème de la malédiction retournée en bénédiction est 
en premier lieu, nous soulignons comment Philon, au travers du décodage symbolique de la figure du devin biblique Balaam, récuse la mantique technique; en second lieu, nous analysons la récupération philosophique de la mantique au travers de la théorie de l'interprétation des songes en De Somniis, II, 1-5. Encore nous faut-il d'entrée de jeu souligner le caractère nécessairement lacunaire de notre étude. En effet, l'étude exégétique et allégorique ${ }^{2}$ utilisée par l'Alexandrin comme méthodologie philosophique de décryptage du texte biblique de la Septante provoque un véritable éclatement de chaque texte en une série de concepts s'interpénétrant de manière très complexe. Il nous a donc fallu écarter du présent travail des développements pourtant indispensables à une intelligence exhaustive de la pensée philonienne en matière d'oracles et de mantique. Les notions clefs de prophétie, d'angélisme, de puissances, de vertu, de sacrifice, de mystères, de prêtrise ne seront qu'évoquées sans être développées. Elles doivent cependant rester présentes à l'esprit si l'on veut percevoir de façon cohérente la théorie de l'art divinatoire élaborée par l'Alexandrin. Les occurrences d'apparition des termes relevant du vocabulaire spécifique de l'oracle et de la mantique dans l'œuvre de Philon sont à la fois

longuement développé, Dieu seul possédant le pouvoir d'inverser le sens des mots. On notera que Balaam est accusé d'avoir accepté pour de l'argent de s'opposer au peuple élu et d'être un "prophète malheureux» (J. CAZEAUX, De Migratione, Ed. Lyon, op. cit., p. 165). Dans ce dernier passage, Philon souligne qu'il est d'usage de dire que Balaam a maudit et non béni l'armée des hébreux, inversant ainsi le sens du texte; De Specialibus Legibus, 48-49, sur la distinction entre la fausse divination et la prophétie inspirée par Dieu lui-même. Il est enfin bon de souligner que dans la Bible en Samuel, I, 28, Saül ordonne que tous les devins soient chassés d'Israël, mais commet la faute de consulter la nécromancienne d'Andor. Philon ne cite pas le texte. Ce climat d'hostilité aux devins est présent tant dans la tradition biblique que dans l'Empire à l'époque même où vivait Philon (É. BRÉHIER, Les idées philosophiques et religieuses de Philon d'Alexandrie, Paris, 1950, p.180-181). Le thème de la mantique était donc brûlant à l'époque de Philon.

2 Nous ne jugeons pas utile d'effectuer une distinction entre commentaire de la Loi et exégèse du texte biblique, nous rangeant à l'avis de V. NIKIPROWETZKY, in Le Commentaire de l'Ecriture chez Philon d'Alexandrie, Prolégomenes à une étude de Philon, p. 241-242, Leiden, Brill, 1977. 
précises et nombreuses ${ }^{3}$. Émile Bréhier, dans son ouvrage bien connu Les idées philosophiques et religieuses de Philon d'Alexandrie, dans le chapitre «Extase et prophétie», procédait déjà à un relevé du vocabulaire philonien en matière de mantique. Il convient naturellement d'ajouter à cette longue liste le vocabulaire inhérent à l'interprétation des songes auxquels Philon a consacré deux livres.

\section{Balaam ou de la divination technique}

La figure du devin Balaam est présentée par Philon comme symbole de la divination technique. En De Vita Mosis, I, Philon procède à une étude exégétique et allégorique du Livre des Nombres, 22-24 de la Septante. Deux remarques préliminaires doivent être formulées sur ce passage où Balaam est invité à se rendre auprès de Balaq, fils de Séphor, afin de maudire l'armée d'Israël. D'une part, le texte concerné présente déjà dans l'original hébraïque des contradictions internes significatives ${ }^{4}$. Il résulte certainement d'une compilation d'au moins deux sources. Philon use des imprécisions relatives à la tectonique du texte pour procéder à un commentaire allégorique extrêmement original. D'autre part, et corrélativement au premier point, il faut observer qu'une lecture synoptique du texte biblique et du commentaire philonien permet d'inférer qu'une grande liberté narrative et interprétative est prise par Philon en regard de l'original. Balaam est d'emblée présenté par l'Alexandrin comme un devin de première force. "Or en ce temps-là, nous dit Philon dans le De Vita Mosis, il y avait un homme célèbre dans l'art oraculaire qui habitait la Mésopotamie : il avait été initié à toutes sortes d'oracles et provoquait l'admiration surtout dans l'art des augures, ayant souvent et à beaucoup de gens annoncé des choses incroyables et importantes" ${ }^{5}$. Les qualités de Balaam en matière divinatoires sont attestées plus avant dans le texte. Entre autres

3 BRÉHIER relève : mantikè, pour l'art oraculaire; oiônoskopiai pour les augures; teratoskopôs pour l'examen des prodiges; thutas pour les aruspices; katartas pour les purificateurs (nous excluons ici tout le vocabulaire relatif au sacrifice qui n'entre pas directement dans notre propos); les procédés magiques relèvent de la magikè sophisteia et nous pouvons ajouter les termes terateuomai (désignant les prodiges, les choses extraordinaires) ainsi que la kakotechnia désignant la techné négative des devins techniques.

4 Voir le commentaire du passage développé en La Bible, La Pléiade, Nombres, 22-24.

5 De Vita Mosis, I, 264, op. cit. La présente traduction est de Roger Arnaldez, Claude Mondésert, Jean Pouilloux et Pierre Savinel. 
qualités, Balaam prédit des pluies en pleine sécheresse, des sécheresses en période de pluies, des fécondités alors que rien ne les laissait présager et vice versa, des crues et des baisses des eaux, la guérison de maladies et bien d'autres prodiges. Cet ensemble de prévisions, que l'on notera plus précisément relatives à la connaissance de la physis, permettent à Balaam d'acquérir une réputation flatteuse dans l'art des oracles et de la divination. Après la victoire des Hébreux sur les Amorréens, Balaq, fils de Séphor, roi d'une cité voisine, s'inquiète de la présence des Hébreux aux frontières de son royaume. Désireux de se concilier les dieux avant que d'affronter des adversaires aussi redoutables, Balaq «se tourna donc du côté des présages et des oracles» ${ }^{6}$. Son objectif est de faire maudire l'armée ennemie avant de livrer bataille. Balaq fait alors appel à l'autorité de Balaam. Alors que jusqu'ici la description des pouvoirs divinatoires et de l'autorité de Balaam semble accréditée par Philon, la suite du commentaire ternit singulièrement l'image du devin. Tout d'abord Balaam joue de son autorité et de sa réputation pour tirer profit de Balaq7; plus grave encore, il feint d'être visité par la divinité, alors qu'il s'agit tout simplement d'un subterfuge dans le but d'augmenter ses honoraires. À la manière des Sophistes ${ }^{8}$, il tire profit de son savoir dans le but d'en tirer un avantage matériel. Il présente d'ailleurs un autre point commun avec "la race des magiciens" que nous dépeignait déjà Platon, puisqu'il fait également preuve d'un grand orgueil. Le commentaire de Philon diverge tant de la Septante que de la Bible hébraïque, puisqu'il n'est jamais fait allusion à une duplicité quelconque dans le texte dont Philon s'inspire pour développer son commentaire exégético-allégorique du livre des Nombres. Au contraire, une lecture attentive du texte original permet de conclure que Balaam éprouve un grand respect pour le Dieu d'Israël. Toutefois, comme nous l'avons souligné, il existe des incohérences dans le texte. Ainsi, lorsque Balaam est prié à la seconde demande des ambassadeurs de Balaq de se rendre auprès de l'armée du Roi, Dieu lui accorde son autorisation ${ }^{9}$. Deux versets plus loin, le Seigneur s'en irrite fortement ${ }^{10}$. Cette première contradiction interne permet de conjecturer l'existence de deux sources antérieures à la

6 De Vita Mosis, I, 263, op. cit.

7 Sur la cupidité de Balaam, voir plus spécifiquement De Cherubim, 32-33 et le De Migratione Abrahami, 113-115.

8 Balaam est explicitement defini comme un Sophiste en Quod Deterius, 71.

9 Nombres, 22 (20).

10 Nombres, 22 (22). 
rédaction du texte. Autre contradiction, les deux serviteurs dont nous parle le texte en 22 (22) ont disparu dans la suite du texte, notamment lors de l'apparition de l'Ange à Balaam. En 22 (24), le devin reconnaît implicitement avoir péché alors que le texte ne fait mention d'aucune faute commise par Balaam au préalable. Ce flottement du texte biblique, Philon le met à profit pour présenter un Balaam cohérent en regard de son système de pensée ${ }^{11}$. Une vision négative du devin qui s'accorde en tous points avec sa hiérarchisation des différents modes de connaissance et partant de vertus. D'un point de vue plus précisément en relation avec la divination, Balaam reste aveugle, tandis que sa monture alogos, "voyait depuis longtemps devant lui une vision qui l'épouvantait»12. L'apparition angélique se montre très sévère pour le devin "dans le but, nous dit Philon, de lui prouver sa vilenie et son néant» ${ }^{13}$. Philon dans le Quod Deus sit immutabilis commente le même passage en précisant que l'Ange est le Logos divin provoquant le déluge des passions dans l'âme égarée de Balaam ${ }^{14}$. L'ange précise la façon dont Balaam rendra l'oracle lorsqu'il comparaîtra devant Balaq. Son discours nous semble tout à fait représentatif de la théorie philonienne en matière de divination. "Avance, dit le Logos, sur la route où tu te hâtes, tu ne serviras à rien, puisque c'est moi qui te souffle ce qu'il faut dire, sans l'intervention de ton intelligence, c'est moi qui recouvre l'organe de ta voix et c'est moi qui tiendrai les rênes de ta parole et ferai chaque révélation par ta bouche, sans que tu comprennes» 15 . La vanité de Balaam s'en trouve ainsi vigoureusement stigmatisée. La véritable erreur de Balaam est d'appuyer son art divinatoire sur la technique et non sur la parole divine. En refusant de se laisser investir par le divin, il perd la dimension authentique censée inspirer le véritable discours divinatoire et oraculaire. Si Philon perçoit de manière négative l'aventure du devin, c'est que son comportement est comparable à celui du Sophiste et du Chaldéen. Tout comme Tharé et Abraham, Balaam

11 Nous entendons par là en regard de la hiérarchisation des vertus et des personnages bibliques que symboliquement Philon leur attribue.

12 De Vita Mosis, I, 272, op. cit. L'âne de Balaam semble avoir ici un rôle positif, cependant en De Cherubim l'ânesse de Balaam, expressément nommée, figure symboliquement les passions sans raison (logos) guidant l'homme "frivole". «... l'ânesse, la déraison prise comme principe de vie que montre tout insensé». (traduction de Jean Gorez, De Cherubim, op. cit.). Dans la suite du texte, Philon stigmatise ceux qui font profession d'amasser de l'argent.

13 De Vita Mosis, 273.

14 Quod Deus Sit Immutabilis, 181.

15 De Vita Mosis, 274. 
vient de la Mésopotamie, il est donc d'origine chaldéenne. Le Chaldéen ${ }^{16}$, pour Philon, est la figure par excellence de la connaissance empirique, sensible. Il s'attache à l'observation du sensible et aux manifestations des phénomènes sans comprendre que l'objet réel de la pensée philosophique se trouve sur un autre registre de la connaissance. Ceci lui interdit de progresser sur la voie de la sagesse. Cette figure de l'homme toujours en quête de la connaissance "scientifique»17, Philon l'apparente à la vaine recherche du sophiste en matière linguistique et à son relativisme philosophique. L'Alexandrin les renvoie dos à dos, considérant qu'ils se trompent tous deux d'objet de connaissance. La mantique technique serait donc récusée en raison de sa proximité méthodologique avec l'empirisme, ce genre de connaissance étant par essence inférieur à la démarche contemplative. Pour surpasser ce blocage, Balaam aurait dû, à la manière de Socrate ou de Tharé ${ }^{18}$ - que Philon qualifie de Socrate biblique - exercer son intelligence à l'approfondissement de sa propre nature; or, à la fin du texte biblique, il est précisé que Balaam s'en retourna vers son pays, ce qui signifie allégoriquement pour l'Alexandrin qu'il refusa de changer de mode de $v^{1}{ }^{19}$. Ce type d'homme laisse de côté toute la partie non rationnelle de l'âme, n'usant que de l'intellect et du raisonnement.

Le passage du devin par l'apprentissage d'une forme de mantique technique explicitement assimilée à une observation rationnelle du monde et à l'emploi méthodologique de la raison n'est pas exclue dans le propos de l'Alexandrin. Le choix de Dieu se portant sur Balaam afin

16 Sur le Chaldéen dans l'œuvre de Philon : voir Wolfson, Philo, T. II, Harvard, Mass., 1948, p. 79 sur les preuves de l'existence de Dieu et T. I, sur une comparaison entre les arguments attribués par Philon aux Chaldéens sur la nature de Dieu et la critique des Stoïciens auxquels en fait s'adresse Philon. Voir également l'analyse de BRÉHIER, Les idées ..., op. cit., p. 165 sur le rapport avec l'astronomie. Il faut noter que le thème des Chaldéens apparaît dans 11 traités distincts chez Philon. Philon s'oppose essentiellement au travers de ce symbole à l'astrologie comme science divinatoire et à la divinisation des éléments.

17 Il s'agit naturellement de la "science" au sens antique.

18 Sur l'interprétation symbolique de Tharé, voir De Somniis, 47-60; sur Tharé "Socrate biblique", le passage suivant du De Somniis : "c'est sur ce comportement que les Hébreux ont mis sous le nom de Tharé et les Grecs de Socrate. Les Grecs affirment en effet que Socrate a vieilli dans une recherche toujours plus pénétrante au sujet du "connais-toi toi-même», n'ayant d'autre philosophie que la connaissance de soi» (De Somniis, traduction Pierre Savinel, Ed. du Cerf, op. cit., p. 47-49).

19 Nombres, 24 (25), non commenté par Philon, à rapprocher toutefois du thème de Tharé et de Haran comme lieu des passions. 
qu'il rende un juste oracle sur l'armée des Hébreux tend à valider l'hypothèse que la mantique technique ne serait qu'une étape propédeutique permettant de déboucher sur la véritable mantique inspirée.

\section{La divination inspirée}

Dans De Somniis, $\mathrm{II}^{20}$, Philon théorise les trois grands types de divination susceptibles de dépasser la mantique technique. Toutes trois s'appliquent à l'interprétation des songes. Nous les présentons ici en ordre qualitatif croissant. Le genre mineur est symbolisé selon Philon par la figure de $\mathrm{Joseph}^{21}$, incarnant à la fois un modèle de bon politique et un genre mineur de la vertu. Les songes interprétés par Joseph sont célèbres par leur opacité et nécessitent un devin spécialisé dans l'art d'interpréter ce type d'oracle divin. En fait, le degré qualitatif de l'interprétation est naturellement en rapport avec le degré de vertu atteint par le devin. "Les apparitions de cette catégorie, précise Philon, plus obscures (que les autres), le sont en raison de la densité de l'énigme qu'elles renferment, elles exigent la science onirocritique»22. Selon Philon, le bon politique doit posséder cette qualité pour diriger de façon efficace le troupeau humain. De façon plus précise, "les rêves de cette catégorie se produisent lorsque pendant le sommeil, l'âme, sortant d'elle-même et se mettant en mouvement, entre dans les transes des Corybantes et, possédée grâce à sa faculté de divination, rend des oracles sur l'avenir» 23 . On remarquera que lorsque Philon décrit l'enthousiasme saisissant l'âme au moment du songe, il use d'une terminologie empruntée à la tradition grecque; de plus, ce type de rêve particulièrement complexe est encore fruit de l'âme humaine elle-

20 De Somniis, II, 1-7.

21 La figure de Joseph intervient de façon contradictoire dans l'œuvre de Philon d'Alexandrie. Il symbolise la figure du politique et partant les imperfections qui sont inhérentes à ce genre de vie. Les principaux traités où la figure de Joseph est étudiée par Philon sont : De Iosepho, Legum Allegoriae, III, Quod deterius, De migratione Abrahami, De mutatione nominum, De Somniis, I. Nous renvoyons à WoLFSON en ce qui concerne la divination, Philo, op. cit., T. II, p. 57. WoLFson y souligne la proximite de la pensée de Philon non seulement avec la Bible mais aussi avec Posidonius d'Apamée. Voir également les conclusions du même chapitre «Knowledge and Prophecy" p. 59-72. Sur le rôle politique de Joseph, cf. le texte de Pierre SAvinel en introduction au De Iosepho; voir également E.R. GOODENOUGH, The Politics of Philo Judaeus, Hildesheim, 1967, p. 21-33; 46-63.

22 De Somniis, II, 4 , op. cit., trad. P. Savinel.

23 Ibid., 1. 
même et donc nécessite quant à son décodage un type de technique : la science onirocritique. Philon ne récuse pas cette techné d'un genre particulier, celle-ci ne s'exerçant sur aucun support matériel concret et la technique en elle-même n'étant qu'une étape. La première catégorie interprétative a donc pour objet des rêves produits par l'âme humaine (en l'occurrence celle du Pharaon); les devins susceptibles d'éclairer l'auteur du rêve sur sa propre vision participent au premier genre de vertu : la vertu politique. Il faut donc qu'il y ait adéquation entre le rêve et celui qui le décrypte pour que le message soit clairement perçu. Paradoxalement, nous le verrons, ce type de rêve est d'apparence très complexe et les devins qui les interprètent paraissent hommes de grand savoir. Deux catégories de rêves doivent encore être interprétés, et avec eux commence la nécessité d'une mantique inspirée. Tout d'abord ceux qui sont directement inspirés par notre esprit et qui permettent de "prédire un grand nombre d'événements à venir»"24. Cette catégorie est en fait inspirée à l'homme qui en est digne par l'âme du monde ${ }^{25}$. On notera que, dans la majorité des traités où cette puissance apparaît, elle est identifiée au Logos divin. Nous avons donc affaire ici au type de visions prophétiques qui inspira Balaam. Ce type de songe, nous dit Philon, est à rapprocher de celui de Jacob. Il s'agit d'une énigme facile à décoder pour "ceux doués d'une vue perçante». Cette catégorie s'avère d'autant plus importante qu'elle a trait au Logos et que l'on sait l'espace fondamental et focal occupé par cette première puissance dans le système philosophique de l'auteur du Legum Allegoriae. Il n'est pas indifférent pour notre propos de constater que la vision de Jacob telle que l'interprète allégoriquement Philon est celle de la montée de l'âme vers Dieu par la médiation des puissances intelligibles. Il s'agit donc d'un type de vision directement en rapport avec le monde des idées. Ces visions ont pour objet le progrès dans la chose philosophique et

24 Ibid., 2.

25 Sur le concept d'âme du Monde chez Philon, nous renvoyons principalement au Quis Rerum Divinarum heres sit et plus spécifiquement au très riche commentaire de M. HARL, qui en introduction à la traduction du traité (Ed. Cerf, op. cit., p. 77-78) développe de manière très claire les rapprochements que l'on peut effectuer avec le Timée de Platon. On peut en effet rapprocher la théorie philonienne du Logos-diviseur et lien de l'univers avec le concept d'Ame du Monde dans le Timée, le Logos Timeus doit d'une part être étudié en parallèle avec les concepts de Même et d'Autre chez Platon, ainsi qu'avec le concept stoïcien de feu. On notera en plus que le vocabulaire employé relève de la sémantique inhérente à la pratique sacrificielle et aux fonctions de prêtrise. Margueritte HARL ne formule cependant aucune conclusion définitive à propos de ces considérations. 
nécessitent un interprète prédisposé naturellement à ces matières, rôle qui convient très bien à Jacob qualifié par Philon d'Athlète de la vertu. C'est par une connaissance parfaite du genre philosophique et sa pratique sans faille que ce type de vision peut être décrypté. Ce genre qu'il faut distinguer de la mantique technique puisqu'elle s'appuie sur un support méthodologique différent - la connaissance philosophique et non celle de la physis - ne peut s'acquérir qu'avec le concours de la raison divine : le Logos. La dernière catégorie de rêves est directement inspirée par Dieu. Ces rêves ont pour fonction de faire la clarté sur les choses intelligibles, opaques pour l'âme humaine mais claires pour la divinité. Le philosophe devient alors comme Moïse le «devin inspiré» qui, tout entier investi par la divinité, devenu alogos comme l'âne de Balaam, abandonne son âme à la divinité et obtient en retour la lumière du monde intelligible. Balaam se trouve donc au terme d'un premier cheminement propice à une compréhension plus nette de la divinité, puisqu'il est aidé par le Logos divin. Là réside sa faute aux yeux de Philon puisqu'il refusa de répondre à cet appel.

Nous pourrions synthétiser la progression du bon devin de la manière suivante : d'abord il s'agira au moyen de la mantique technique d'augurer sur base d'une vision "scientifique" du monde. Les visions issues de ce type de pratique, nous l'avons vu dans le cas de Balaam, s'apparentent à l'observation et à la connaissance d'un genre inférieur. Ensuite, le devin sans support concret saura décrypter les messages opaques transmis par l'âme, et en déchiffrer le secret. Ce type de divination nécessite des prédispositions à une vertu d'ordre pratique, comme celle de Joseph, sans pour autant impliquer une inspiration extérieure. Il s'agit en fait d'une divination sagesse pratique, utile à la conduite de la vie. Le Pharaon rend justice sur le cas de l'Échanson et du Panetier, il prend les précautions nécessaires au bon fonctionnement de son empire. Bref, ces rêves d'ordre pratique sont le fruit d'une bonne intelligence politique du monde mais non métaphysique. Le troisième type de rêve a pour objet le monde intelligible mais sous forme d'énigme simple que la connaissance seule ne peut justifier. Inspiré par le divin Logos, il s'agit de rêves permettant une vision globale et partant philosophique de l'harmonie du monde. Il est donc naturel que ces rêves soient inspirés par le Logos divin, fonction unificatrice et globalisante par excellence dans le système philosophique de Philon. Il est nécessaire que cette catégorie de songes soit décodée par un homme présentant des aptitudes à la vertu et au travail philosophique.

La dernière catégorie, de loin la plus complexe, nécessite un total dépouillement et, en une démarche quasi-mystique, permet la compré- 
hension de ce qui est entièrement voilé à la face des hommes. Ces rêves ne sont prodigués qu'à ceux qui ont atteint le plus haut degré de vertu humaine possible et viennent comme le complément philosophique d'origine divine qui seul peut mener à la perfection. Le philosophe se transforme ainsi en une sorte d'anti-philosophe alogos comme l'âne du devin Balaam, tout entier livré à Dieu et dont la voix permet l'écoulement sans obstacle de la parole divine. On songe ici à la figure de Moïse dont Philon fait le modèle philosophique par essence.

Un beau passage du De Specialibus Legibus résume très bien la position de Philon en matière de divination et d'oracles. Philon y stigmatise avec virulence «les déchiffreurs de prodiges, les augures, les haruspices et tous les autres experts en divination, dont les activités consistent, à franchement parler, en une science de malheur savamment concertée, contrefaçon de la possession et de la prophétie divines» 26 . Il faut donc, comme nous l'avons souligné, considérer deux types de divination, l'une relevant de la technique que Philon rejette et l'autre de la possession de l'âme par Dieu que Philon déclare conforme à la vraie philosophie. Ce second type de devin est ici nommé Prophète. Cette distinction sémantique opérée par Philon lui permet alors d'attaquer la divination de manière plus violente. "Quant aux adeptes de la divination, cette science de faussaires et de bouffons, chacun d'eux assigne à ses conjectures et à ses inductions un rang inadéquat, celui de la vérité (alètheia) et n'a pas de mal à circonvenir les caractères influençables» 27 . Les hommes qui pratiquent ce type de divination sont appelés faux prophètes par Moïse (le Législateur). À ce genre de divination, Philon oppose la prophétie - divination inspirée comme nous l'avons vu -, le véritable devin n'étant autre que le Prophète. Sa définition correspond d'ailleurs à celle du devin inspiré puisque Philon précise «... le Prophète ne publie absolument rien de son cru, mais il est l'interprète d'un autre personnage qui lui souffle toutes les paroles qu'il articule, au moment même où l'inspiration le saisit et où il perd la conscience de lui-même, du fait que la raison émigre et abandonne la citadelle de l'âme cependant que l'Esprit divin visite et habite celle-ci et qu'il fait retentir et résonner de l'intérieur toute l'instrumentation vocale pour manifester clairement ce qu'il prédit» 28 . L'aventure de Balaam et la vision négative que Philon nous en donne s'en trouve donc éclairée. Le devin, en dépit de l'exemple divin, refusa de franchir la

26 De Specialibus Legibus, IV, 48-49 (Ed. du Cerf, op. cit., Trad. André Mosès).

27 Ibid., 50.

28 Ibid., 49. 
barrière qui le séparait de la véritable divination. Homme de l'extérieur et des signes matériels, Balaam avait négligé d'examiner la révélation du dedans.

Pour conclure, Philon effectue une critique sévère de la mantique technique. Tout en concédant son utilité au niveau matériel, il stigmatise son côté réducteur au niveau spirituel. Le Devin usant de la mantikè technè est comparable au Sophiste et au Chaldéen, qui par leur savoir apparent s'écartent du véritable savoir.

Le Devin véritablement inspiré est identifiable au véritable philosophe ou prophète, au point de poser une question ultime à laquelle nous n'oserions répondre : la mantique dans le sens où les Grecs l'entendaient ainsi remaniée, catégorisée, dépouillée et transmise aux Pères par l'auteur des Allégories possède-t-elle encore le statut d'un art oraculaire et divinatoire ? Ne perdait-elle pas la spécificité plurivoque de l'héritage polythéiste pour devenir un jouet entre les mains du Philosophe?

Le visiteur de la basilique Saint Andoche à Saulieu peut voir, sur l'un des admirables chapiteaux romans de l'édifice, un petit vieillard, juché sur un âne, le visage figé dans la pierre en une muette expression de stupeur. Bien sûr, c'est Balaam, pour toujours médusé par les conclusions terribles qu'un philosophe d'Alexandrie devait tirer de sa promptitude à servir Dieu.

Université libre de Bruxelles

Baudouin DECHARNEUX

Av. Fr. Roosevelt, 17

B - 1050 BRUXELLES 